\title{
Analysis of the bounds on dark matter models from monojet searches at the LHC
}

\author{
Swasti Belwal* and Manuel Drees ${ }^{\dagger}$ \\ BCTP, Physics Institute, University of Bonn, 53115 Bonn, Germany \\ Jong Soo Kim ${ }^{\ddagger}$ \\ National Institute for Theoretical Physics, School of Physics and Mandelstam Institute for Theoretical Physics, \\ University of the Witwatersrand, 2050 Johannesburg, South Africa
}

(Received 20 October 2017; published 13 September 2018)

\begin{abstract}
We analyze the constraints on models of WIMP dark matter that can be derived from upper bounds on the "monojet" cross section at the LHC. These constraints were originally interpreted in the context of an effective field theory (EFT) where the Standard Model is extended by a dimension-6 operator whose coefficient is $1 / \Lambda^{2}$. We show that combining the $8 \mathrm{TeV}$ data of the ATLAS and CMS collaborations improves the bounds only slightly. We then analyze this final state in the context of simplified models with an $s$-channel mediator. We show that if the decay width of the mediator is small, these simplified models can be accurately modeled by the effective field theory only if the mediator mass is above $5 \mathrm{TeV}$. Finally, we point out that even if the EFT accurately describes the $\mathcal{O}\left(\Lambda^{-2}\right)$ contributions to the matrix element, for values of $\Lambda$ near the current bound it receives significant contributions of order $\Lambda^{-4}$; in the context of simplified models, these correspond to diagrams where two mediators are exchanged. This observation challenges the internal consistency of the EFT description since dimension-8 operators, which would also contribute to $\mathcal{O}\left(\Lambda^{-4}\right)$ to the matrix element, are not included.
\end{abstract}

DOI: $10.1103 /$ PhysRevD.98.055017

\section{INTRODUCTION}

A large number of astrophysical and cosmological observations indicate that most of the matter in the Universe is nonbaryonic [1]. The Standard Model of particle physics (SM) does not contain a particle that could form this "dark matter." Probably the most widely studied candidate particle is a weakly interacting massive particle (WIMP), with mass in the $\mathrm{GeV}$ to $\mathrm{TeV}$ range and with very roughly weak-strength annihilation cross section into SM particles. The latter implies that, within standard cosmology, WIMPs that were in thermal equilibrium have approximately the correct relic density (the so-called "WIMP miracle") $[2,3]$. Moreover, many extensions of the SM that address some of its other shortcomings automatically contain, or easily accommodate, viable WIMP candidates [1,4].

\footnotetext{
swasti@th.physik.uni-bonn.de †drees@th.physik.uni-bonn.de

*jongsoo.kim@tu-dortmund.de
}

Published by the American Physical Society under the terms of the Creative Commons Attribution 4.0 International license. Further distribution of this work must maintain attribution to the author(s) and the published article's title, journal citation, and DOI. Funded by SCOAP ${ }^{3}$.
Another reason for the popularity of WIMPs as dark matter candidates is that there are several distinct methods to search for them, owing to their significant interactions with SM particles. In particular, WIMP interactions with quarks, and hence nucleons and nuclei, allow dark matter WIMPs from the halo of our galaxy to scatter off ordinary matter, depositing a few (tens of) $\mathrm{keV}$ of energy. So-called direct (WIMP) detection experiments search for this signature [1]. The very same interactions should also allow us to produce pairs of WIMPs ${ }^{1}$ at hadron colliders like the LHC. Since WIMPs must be stable on collider timescales and electrically neutral, they escape detection. The production of a WIMP pair must therefore be tagged by the emission of a high $p_{T}$ object recoiling against the WIMP pair, which manifests itself as missing $p_{T}$. The largest rates, and hence the best signals for WIMPs that interact with quarks, result when the WIMPs recoil against a jet, leading to a monojet signal $[5,6]$.

Ambient dark matter WIMPs are nonrelativistic, with typical velocity $v \sim 10^{-3} c$. Hence the maximal momentum exchanged in direct detection experiments is of order $100 \mathrm{MeV}$ (for scattering of a relatively heavy WIMP on

\footnotetext{
${ }^{1}$ Single WIMP production is not possible as the time reversed version of such matrix elements would allow WIMPs to decay, ruling them out as dark matter candidates.
} 
a heavy target nucleus like xenon). Since WIMPs carry neither electric nor color charge, their interaction with quarks must be mediated by one of the neutral massive SM bosons, or by a new particle. In most cases the mass of this mediator is much larger than $100 \mathrm{MeV}$. In this case, direct detection experiments can be analyzed in an effective field theory (EFT) where the mediators have been integrated out, giving rise to higher-dimensional operators. This simplifies the analysis since EFTs often have fewer free parameters than complete models; moreover, several models may lead to the same EFT, and can thus be treated simultaneously.

Monojet signals have therefore also been analyzed in terms of an EFT, both by theorists [5,6] and by the LHC collaborations $[7,8]$. Clearly this can be expected to accurately reproduce the results obtained in a renormalizable theory only if the momentum flow through the mediator is (much) smaller than the mass of this mediator, as we just saw for WIMP scattering off baryonic matter. The most sensitive search region for the $8 \mathrm{TeV}$ data requires missing transverse momentum of about $500 \mathrm{GeV}$. One may then conclude that the EFT description should work if the mediator mass is (well) above $500 \mathrm{GeV}$. In fact, this estimate is not that far off for $t$-channel mediators $[9,10]$. However, we will see below that the exchange of narrow $s$-channel mediators can be accurately modeled by an EFT only for mediator masses above $5 \mathrm{TeV}$. In this case the experimental bounds on $\Lambda$, which are around $1 \mathrm{TeV}$, can be saturated only if the mediator's couplings to quarks and/or WIMPs are considerably above 1, i.e., for strongly interacting theories.

The question of whether an EFT can accurately describe the LHC signatures of a simplified, or-better yet-a UVcomplete model with an $s$-channel mediator has been investigated before. For example, in [11] it was pointed out that the EFT prediction for the partonic cross section for the production of a pair of WIMPs violates unitarity at sufficiently high energies. The requirement that this does not happen at energies accessible at the LHC gives a stronger bound on $\Lambda$ than monojet searches do. However, this does not exclude the possibility that a UV-complete model can be described by an EFT at energies relevant for the monojet search, but regularizes the WIMP pair production cross section at yet higher energies.

Moreover, it was pointed out early on $[5,12,13]$ that the true LHC constraint on simplified models can differ greatly from the limit derived in the corresponding EFT. The EFT will overestimate the true bound on $\Lambda$, possibly by several orders of magnitude, if the mediator is light and cannot decay into two WIMPs; conversely, the EFT will underestimate the true bound if the mediator is not very heavy and can decay into two WIMPs.

It is clear that UV-complete models with a relatively light mediator cannot be described reliably by an EFT as far as monojet searches at the LHC are concerned. On the other hand, it has been pointed out that a reliable lower bound on
$\Lambda$ can be derived by restricting the calculation of the signal cross section to kinematical configurations where the momentum flowing through the propagator of the mediator is (well) below the mediator's mass [14-16]. These bounds are reliable in the sense that any theory that can be mapped onto an EFT which violates the LHC constraints derived in this manner is indeed excluded by the monojet searches. However, the bound one derives in this manner on UVcomplete models is often far weaker than the true bound from LHC data. For example, even the sophisticated statistical analysis of Ref. [17] finds a bound on $\Lambda$ which is four times weaker than the corresponding bound derived in the framework of a simplified model with a dynamical $s$-channel mediator, even for a mediator mass of $2 \mathrm{TeV}$. This is not very surprising, since the cut on momentum flow excludes events where the mediator is approximately on shell; for a mediator mass around $2 \mathrm{TeV}$ such events dominate the total signal cross section. This means that the EFT result derived in this manner in many cases cannot be used to check whether a given simplified model is compatible with monojet searches at the LHC or not. In other words, satisfying this version of the EFT bound, which is weaker than the EFT bound derived by the experiments themselves, is a necessary, but not a sufficient condition on UV-complete theories. However, this still allows for the possibility that at least some subclass of simplified models can indeed be described accurately by an EFT as far as monojet searches at the LHC are concerned.

Note that in most of these earlier analyses the width of the mediator was fixed to be some fraction of its mass. This may be acceptable in a simplified model, but is definitely not correct in a complete theory where the width is a derived quantity. In fact, it had been pointed out in [18] that a simplified model with $s$-channel mediator that comes close to saturating current LHC constraints can be naively described by an EFT only for regions of parameter space where the computed width of the mediator is larger than its mass. We will see in Sec. V that this implies that no weakly coupled model with a dynamical $s$-channel mediator, whose width is computed from its mass and its couplings, can be described by an EFT for parameters that can be probed at the LHC.

Moreover, we find that even in the EFT limit, which may describe strongly coupled theories, some contributions to the matrix element for WIMP production that are of order $\Lambda^{-4}$ are sizable. This means that an accurate EFT treatment would need to include dimension- 8 operators, thereby introducing (many) new parameters, spoiling the main advantage of EFTs. Simply ignoring all these $\Lambda^{-4}$ terms, which seems to have been standard practice in the experimental analyses, thus means that the EFT as applied to monojet searches does not accurately describe any renormalizable theory with an $s$-channel mediator, if the scale $\Lambda$ is near the experimental lower bound.

The reminder of this paper is organized as follows. In the next section we discuss the theoretical setup, followed by a 
description of the technical details of our analysis in Sec. III. In Sec. IV we show that combining $8 \mathrm{TeV}$ data from the ATLAS and CMS collaborations only slightly strengthens the lower bound on the EFT scale $\Lambda$. Section V contains a comparison of EFT results with results obtained in simplified models with pseudoscalar or axial vector $s$-channel mediators. The impact of $\mathcal{O}\left(\Lambda^{-4}\right)$ contributions to the matrix elements are discussed in Sec. VI. Finally, we summarize our results and draw some conclusions in Sec. VII.

\section{THEORETICAL FRAMEWORK}

We are interested in theories of WIMP dark matter interacting with quarks. Such theories can be tested both via direct WIMP searches and via searches for events with missing transverse momentum at hadron colliders such as the LHC. For definiteness we assume that the WIMP is a Dirac fermion $\chi$. Models with Majorana fermions would lead to very similar results for the interactions we consider. Models with a (real or complex) scalar WIMP obviously do not lead to spin-dependent contributions to the matrix elements for WIMP-nucleon scattering; such WIMPs cannot interact with protons via the exchange of pseudoscalar or axial vector mediators. UV-complete models where the WIMPs carry a spin of one unit are a bit more complicated, without adding anything fundamentally new to our study.

An effective field theory (EFT) describing the interactions of $\chi$ with standard quarks $q$ can then be written as [5]

$$
\mathcal{L}_{\mathrm{EFT}}=\sum_{\Gamma} \frac{1}{\Lambda_{\Gamma}^{2}} \bar{\chi} \Gamma \chi \bar{q} \Gamma q
$$

Here $\Gamma \in\left\{1, i \gamma_{5}, \gamma_{\mu}, \gamma_{\mu} \gamma_{5}, \sigma_{\mu \nu}\right\}$ for scalar, pseudoscalar, vector, axial vector, and tensor interactions, respectively. In principle WIMPs could couple to different quarks with different strengths; i.e., the parameters $\Lambda_{\Gamma}$ could depend on the flavor of $q$ as well. However, we will assume that all quarks couple with equal strength, or not at all, to the WIMPs [i.e., some heavy quarks may not appear in the effective Lagrangian of Eq. (1)]. Moreover, in order to further simplify the analysis, we will assume that only one of the operators in Eq. (1) is present; i.e., all but one of the $\Lambda_{\Gamma}$ will be sent to infinity.

The effective Lagrangian of Eq. (1) allows us to describe the production of a $\chi \bar{\chi}$ pair in $q \bar{q}$ annihilation. If $\chi$ is to be a dark matter particle, it should be electrically and color neutral, and stable on collider timescales. Such a particle will traverse a LHC detector without trace. Inclusive $\chi \bar{\chi}$ pair production thus looks like producing "nothing," as far as the LHC detectors are concerned. Clearly this is not a viable signature.

The signature becomes viable if "nothing" recoils against a high- $p_{T}$ object. The largest cross section, and strongest bound, results when this object is a jet, which can result from the emission of a single high- $p_{T}$ parton. Since

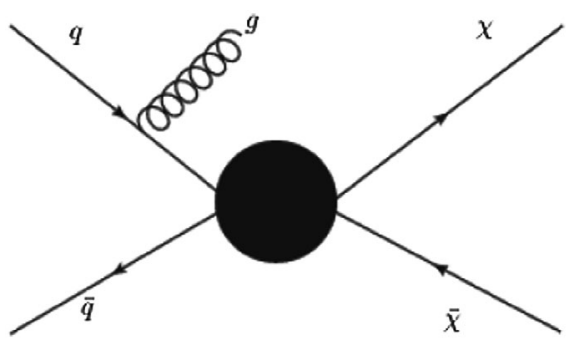

FIG. 1. A Feynman diagram giving rise to a monojet event when the WIMPs $(\chi, \bar{\chi})$ escape detection. The blob stands for one of the four-point EFT interaction vertices of Eq. (1).

the WIMPs escape detection, this leads to the celebrated "monojet" signature, where the event contains a single hard jet, leading to a large amount of missing transverse momentum. A Feynman diagram leading to this signature in the framework of the EFT of Eq. (1) is shown in Fig. 1.

One of the goals of our analysis is to compare predictions derived from the EFT defined by Eq. (1) with those derived from a "simplified model," where $\chi$ interacts with quarks via the exchange of one mediator. Here we focus on $s$-channel mediators for two reasons. First, $t$-channel mediators would need to carry color, whereas $s$-channel mediators do not. The latter can for example be additional gauge or Higgs bosons, which have been widely discussed in the literature for reasons not related to dark matter; such models therefore seem somewhat better motivated than models with $t$-channel mediators. ${ }^{2}$ Secondly, in models with $t$-channel mediators a Fierz transformation is required to bring the effective Lagrangian into the form of Eq. (1), which has also been used by the experimental groups. Hence a simplified model with a $t$-channel mediator will generally produce several terms in the effective Lagrangian simultaneously, thereby complicating the analysis.

Among the $s$-channel mediators, models with a scalar $(C P$-even) or vector mediator will lead to spin-independent contributions to the WIMP-nucleon scattering matrix elements. There are very strong constraints on such interactions from direct dark matter search experiments $[1,20,21]$. Moreover, there is no renormalizable theory with mediators of spin-2, which would lead to tensor interactions. ${ }^{3}$ Hence we focus on pseudoscalar ( $C P$-odd)

\footnotetext{
${ }^{2}$ An exception here is supersymmetry which automatically contains $t$-channel mediators, namely the squarks. However, even the simplest potentially realistic supersymmetric extension of the SM, the minimal supersymmetric standard model (MSSM) [19], also contains $s$-channel mediators, since the WIMPs also couple to $Z$ and neutral Higgs bosons. The MSSM can therefore in general not be described by a simplified model.

${ }^{3}$ Tensor interactions might also originate from the $t$-channel exchange of mediators with lower spin, via Fierz rearrangement; however, this will lead to additional interactions. Pure tensor interactions from the exchange of a single mediator can only be produced if the mediator is a spin-2 particle exchanged in the $s$-channel.
} 
and axial vector mediators. In the subsequent subsections we discuss these two cases in turn.

\section{A. Axial vector mediator}

The general Lagrangian describing the WIMP $\chi$ interacting with quarks $q$ via the exchange of an axial vector (AV) mediator $A$ is

$$
\begin{aligned}
\mathcal{L}_{\mathrm{AV}}= & \mathcal{L}_{\mathrm{SM}}-\frac{1}{4} A^{\mu \nu} A_{\mu \nu}+\frac{1}{2} M_{A}^{2} A^{\mu} A_{\mu}+i \bar{\chi} \gamma^{\mu} \partial_{\mu} \chi \\
& -m_{\chi} \bar{\chi} \chi+g_{\chi A} A_{\mu} \bar{\chi} \gamma^{\mu} \gamma^{5} \chi+\sum_{q} g_{q A} A_{\mu} \bar{q} \gamma^{\mu} \gamma^{5} q .
\end{aligned}
$$

Here, $\mathcal{L}_{\mathrm{SM}}$ stands for the general standard model Lagrangian; $A^{\mu \nu}=\partial^{\mu} A^{\nu}-\partial^{\nu} A^{\mu}$ is the field strength tensor; $g_{q A}$ and $g_{\chi A}$ are the couplings of the mediator to $q$ and $\chi$, respectively; and finally, $M_{A}$ and $m_{\chi}$ are the masses of the mediator and the WIMP, respectively.

We neglect couplings of the mediator to the leptons since they play no direct role in the monojet signature we will investigate. Moreover, there are stringent constraints on such couplings from dilepton resonance searches [22]. As noted above, we take equal couplings of the mediator to all the quark flavors; the Lagrangian (2) then respects SM gauge invariance. When we integrate out the $A$ field, the effective dimension- 6 four-fermion operator corresponding to the Lagrangian of Eq. (2) is

$$
\mathcal{O}_{\mathrm{AV}}^{6}=\frac{1}{\Lambda^{2}}\left(\bar{q} \gamma_{\mu} \gamma_{5} q\right)\left(\bar{\chi} \gamma^{\mu} \gamma_{5} \chi\right)
$$

For a weakly coupled theory, the total decay width $\Gamma_{A}$ of the mediator should be much smaller than its mass. The cutoff scale $\Lambda$ of the effective theory is then

$$
\Lambda=M_{A} / \sqrt{g_{\chi A} g_{q A}} .
$$

If $\Gamma_{A}$ is not negligible, the numerator in Eq. (4) should be replaced by $\left[M_{A}^{2}\left(M_{A}^{2}+\Gamma_{A}^{2}\right)\right]^{1 / 4}$. We will see later that this can cause problems if $M_{A}$ is significantly larger than $\Lambda$.

\section{B. Pseudoscalar mediator}

The case of a real spin- $0 C P$-odd pseudoscalar (PS) mediator $P$ is described by the following Lagrangian:

$$
\begin{aligned}
\mathcal{L}_{\mathrm{PS}}= & \mathcal{L}_{\mathrm{SM}}+\frac{1}{2}\left(\partial_{\mu} P\right)^{2}-\frac{1}{2} M_{P}^{2} P^{2}+i \bar{\chi} \not_{\mu} \chi-m_{\chi} \bar{\chi} \chi \\
& -i g_{\chi P} P \bar{\chi} \gamma^{5} \chi-\sum_{q} i g_{q P} P \bar{q} \gamma^{5} q .
\end{aligned}
$$

Here, $g_{q P}$ and $g_{\chi P}$ are the couplings of the quarks and the WIMP to the mediator, and $M_{P}$ and $m_{\chi}$ are the masses of $P$ and $\chi$, respectively. We again ignore possible couplings of the mediator to leptons.
Note that the couplings in Eq. (5) violate chirality. It is usually assumed that chirality breaking in the SM is governed by the fermion masses. We therefore follow the usual assumption $[5,8]$ by setting the couplings of $P$ to quarks to be proportional to the quark masses:

$$
g_{q P}=g \frac{y_{q}}{\sqrt{2}}
$$

where $y_{q}$ is the SM Yukawa coupling of quark $q$ and $g$ is a real constant. ${ }^{4}$ Integrating out the $P$ field gives the dimension-6 effective operator:

$$
\mathcal{O}_{\mathrm{PS}}^{6}=\frac{m_{q}}{\Lambda^{3}}\left(\bar{q} \gamma_{5} q\right)\left(\bar{\chi} \gamma_{5} \chi\right)
$$

The cutoff scale $\Lambda$, which from Eq. (6) is common for all quarks (with nonvanishing coupling to $P$ ) is given by

$$
\Lambda^{3}=\frac{M_{P}^{2} m_{q}}{g_{q P} g_{\chi P}}=\frac{v M_{P}^{2}}{g g_{\chi P}},
$$

where $v \simeq 246 \mathrm{GeV}$ is the vacuum expectation value of the Higgs field breaking the electroweak gauge symmetry. Here we have again assumed that the theory is weakly coupled, i.e., that the total decay width $\Gamma_{P}$ of the mediator is significantly smaller than its mass, $\Gamma_{P}^{2} \ll M_{P}^{2}$.

It should be noted that simplified models with $s$-channel mediators necessarily also generate dimension- 6 operators of type $(\bar{q} q)\left(\bar{q}^{\prime} q^{\prime}\right)$ (where $q^{\prime}$ and $q$ may be different or equal quarks) and $(\bar{\chi} \chi)(\bar{\chi} \chi)$, with coefficients that are proportional to the square of the mediator's coupling to quarks and WIMPs, respectively. To leading order in an expansion in powers of $\Lambda^{-2}$ these additional operators play no role in the cross section for monojet production; we will nevertheless see below that their contribution can be significant for values of $\Lambda$ near the current lower bound.

\section{ANALYSIS FRAMEWORK}

In this section we describe technical details of our analysis of monojet signals predicted by the models we described in the previous section.

\footnotetext{
${ }^{4}$ The Lagrangian (5) does not respect SM gauge invariance if both $P$ and $\chi$ are $S U(2)$ singlets. One UV completion of our simplified model is a two Higgs doublet model where $P$ is the single physical pseudoscalar state, which should be lighter than the heavy neutral scalar state for $P$ exchange to dominate. The $\chi$ field must then also have an $S U(2)$ doublet component for the $P \bar{\chi} \chi$ coupling to be nonzero. Alternatively one can introduce an SM singlet with pseudoscalar coupling to $\chi$, which can then also be a pure singlet, but with a $C P$-violating mixing with the SM Higgs [23]. $P$ will then have pseudoscalar coupling to $\chi$ but scalar coupling to quarks. The pseudoscalar coupling to $\chi$ suffices to remove spin-independent contributions to $\chi p$ scattering, while the LHC phenomenology does not depend strongly on whether the coupling is scalar or pseudoscalar.
} 
We begin with writing the model files in FeynRules-v2.0 [24]. All the necessary model details for the particles and their couplings, along with the complete Lagrangian, are given as an input to FeynRules-v2.0. The output from FeynRules, describing all the possible interactions in the Universal Feynrules Output format (UFO) [25], is then used as input to MadGraph5-aMC.@.NLO.v2.3.0 [26] for matrix element calculations and event generation. The calculation is done at tree level. We use the MSTW2008LO set of parton densities [27] as implemented in the LHAPDF package [28].

The monojet signal requires the existence of at least one hard jet, but events with at least one additional jet are also accepted. We therefore ask MadGraph to generate events with a $\chi \bar{\chi}$ pair plus one or two hard partons in the final state. These events are passed to PYTHIAv6.4 [29], which is integrated with MadGraph5-aMC.@.NLO.v2.3.0, for showering. Hard parton showering off the $\chi \bar{\chi}$ plus one parton sample of MadGraph events leads to the same final state as $\chi \bar{\chi}$ plus two parton MadGraph events with only relatively soft showering. In order to remedy this double counting we use the MLM matching prescription [30] for jets.

Our cross section will diverge as the $p_{T}$ of the jets goes to zero, as do all cross sections involving jets at hadron colliders. Since the experimental monojet searches use strong cuts on the missing $E_{T}\left(E_{T}\right)$ and on the $p_{T}$ of the hardest jets, we apply parton-level cuts at the MadGraph level of $p_{T}$ of $200 \mathrm{GeV}$ for the leading jet and minimum $E_{T}$ of $300 \mathrm{GeV}$. We use a value of $100 \mathrm{GeV}$ for the xqcut variable, which separates the region of phase space to be populated by showering from that populated by the second hard parton explicitly generated by MadGraph; we checked that varying the value of xqcut by up to a factor of 2 has little impact on the final cross section after all cuts. All other parameters are kept at their default values. Since the rather stiff generator-level cuts ensure that the efficiency for passing the final cuts is not very low, we found it sufficient to generate 50,000 events per point in parameter space.

The final selection cuts for the various monojet signal regions as well as a (simplified) simulation of detector effects are performed with CHECKMATE-v2.0 [31,32] based on DELPHES-v3.0.10 [33] with modified detector cards as well as FASTJET-v3.0.6 [34] for the jet reconstruction. To that end, we implemented the ATLAS [8] and CMS [7] monojet analyses for $\sqrt{s}=8 \mathrm{TeV}$ in CHECKMATE-v2.0 following the prescription of [35]. Briefly, both ATLAS and CMS veto the presence of identified charged leptons, in order to suppress backgrounds from $W+$ jets. Several signal regions are defined, which differ by the lower bound on the missing $E_{T}$; the most sensitive signal regions, which are used to set the final cuts, typically have $E_{T}^{\prime}>400$ or $500 \mathrm{GeV}$.
It is important to note that neither ATLAS nor CMS strictly speaking search for pure monojet events. Vetoing events with a second reconstructed jet would reduce the signal considerably, since many events with $E_{T} \sim 500 \mathrm{GeV}$ produce at least one additional jet from showering. CMS rejects events that have more than two jets with $p_{T}>30 \mathrm{GeV}$; moreover, if there is a second jet, the azimuthal angle between the two jets has to be less than 2.5 radians (or 143 degrees). ATLAS does not impose an explicit upper bound on the number of jets but requires that the $p_{T}$ of the hardest jet be at least $50 \%$ of the total $E_{T}$; moreover, all jets must have an azimuthal angle relative to the missing $p_{T}$ vector of at least 1 radian (57 degrees).

We use the model with an axial vector mediator to validate our analysis chain by comparing our bounds on the scale $\Lambda$ with the bounds derived by the ATLAS and CMS collaborations, for various values of the WIMP mass between $1 \mathrm{GeV}$ and $1 \mathrm{TeV}$. Since we wish to reproduce the EFT limit, we set the mediator mass to $10 \mathrm{TeV}$, and its width to $1 \mathrm{GeV}$ such that Eq. (4) is applicable. For the generation of MadGraph events, we set $N_{\mathrm{NP}}=2$; i.e., we allow only a single exchange of the mediator in the signal process. Results for the $8 \mathrm{TeV}$ analyses are depicted in Fig. 2. We see that we reproduce the experimental lower bound on the scale to better than $10 \%$. From this figure the CMS limit looks more stringent than that of ATLAS. Note, however, that ATLAS quotes a lower bound at 95\% confidence level (C.L.), whereas CMS only requires a C.L. of $90 \%$.

For the early (2015) $\sqrt{s}=13 \mathrm{TeV}$ data, ATLAS and CMS do not use the EFT interpretation of their monojet bounds any more. We instead consider the simplified model analysis of ATLAS [36]. We implemented their signal regions into CHECKMATE-v2.0 and tested our effective model against this search. Although at this increased center-of-mass energy the on-shell production of the mediator is in principle possible, we found that this contribution is negligible because of the very small parton densities at the required large parton energies. Figure 3 shows that the resulting lower bound on the cutoff scale is approximately $100 \mathrm{GeV}$ stronger than that derived from data taken at $\sqrt{s}=8 \mathrm{TeV}$, although the integrated luminosity of the latter data set is about six times larger.

Owing to the factor of $m_{q}$ in the coefficient of the pseudoscalar four-fermion operator [see Eq. (7)], the resulting bound on $\Lambda$ is much weaker than in the axial vector case. For example, even for a light WIMP ATLAS quotes a lower bound of just $40 \mathrm{GeV}$ on $\Lambda$. This is well below the values of even the basic acceptance cuts defining monojet events, making the usefulness of an EFT description in this case a priori unlikely. However, formally our setup can also treat this case, by simultaneously choosing a large pseudoscalar mediator mass, a small mediator width, and very large values for the coefficient $g$ defined in Eq. (6). 

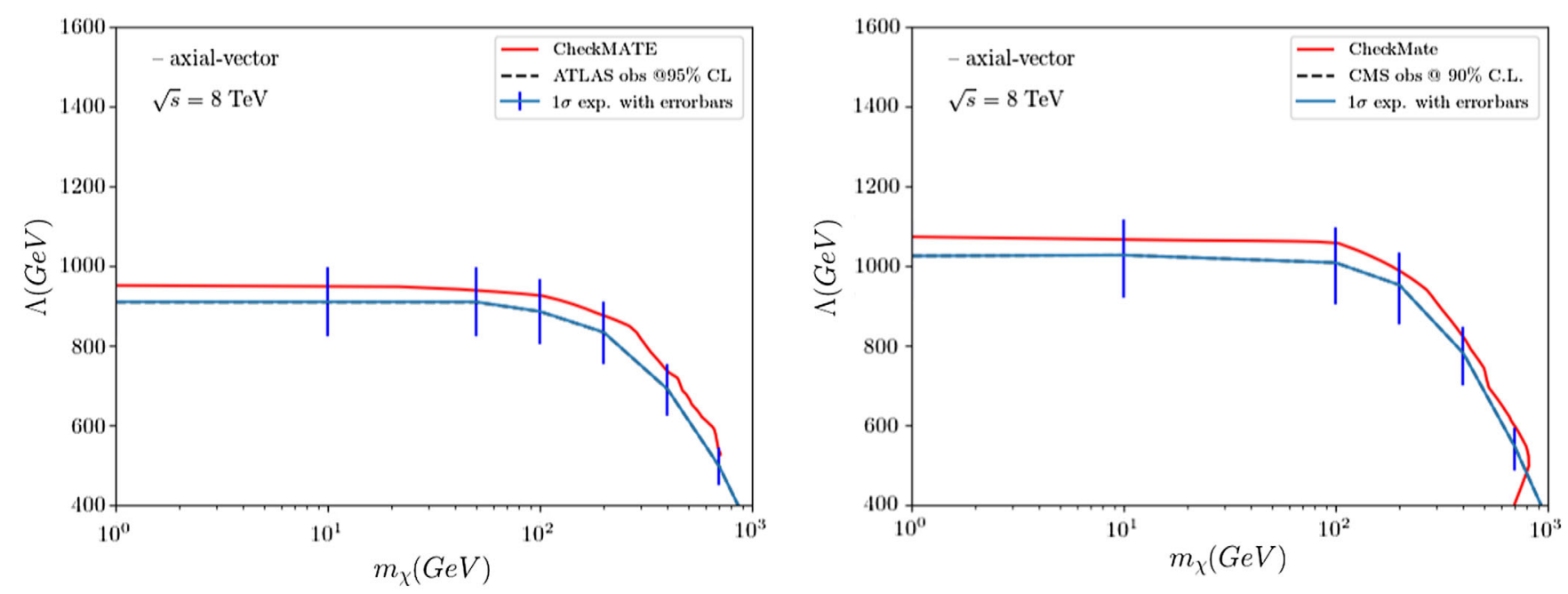

FIG. 2. Our limits on the strength of the axial vector interaction derived using CHECKMATE compared to the ATLAS limits on $\Lambda$ at 95\% C.L. and CMS limits on $\Lambda$ at 90\% C.L. for $\sqrt{s}=8 \mathrm{TeV}$.

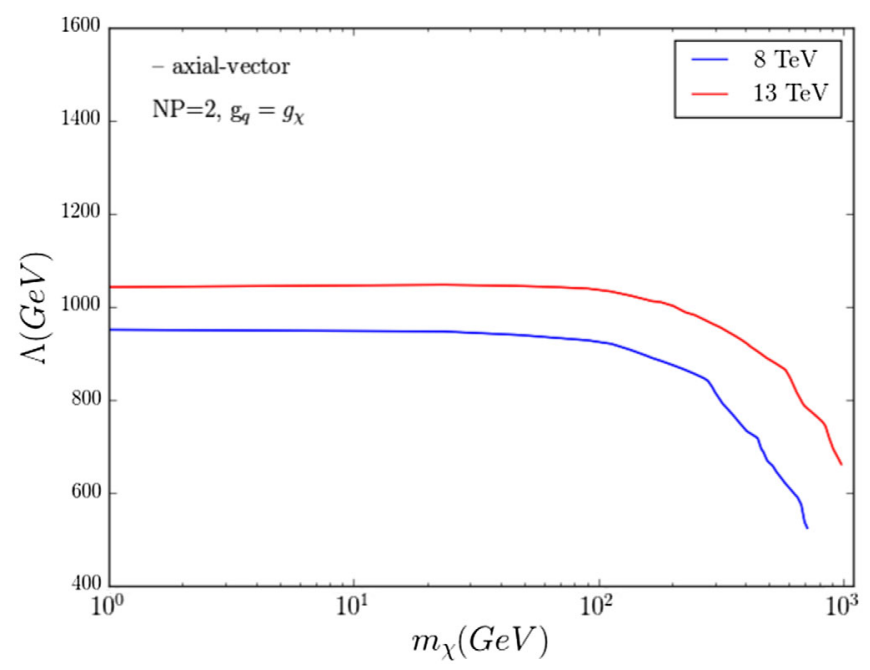

FIG. 3. Bounds on $\Lambda$ for axial vector interaction at $95 \%$ C.L. using ATLAS data taken in 2015 at $\sqrt{s}=13 \mathrm{TeV}$.

\section{COMBINED ANALYSIS}

Since ATLAS and CMS give comparable bounds on the scale $\Lambda$, one expects to obtain stronger limits by combining both data sets. Both ATLAS and CMS use the "confidence level" method to set the limits $[37,38]$. If the errors on the background estimates are not correlated between the experiments it is straightforward to combine the results. The combined total background error will then be the sum in quadrature of the two separate errors. This is probably not a bad approximation since the background estimates are data driven. We use Gaussian statistics for this computation.

To explain how we estimate the combined limits we briefly describe an example of combining ATLAS SR7 $\left(E_{T}>500 \mathrm{GeV}\right)$ [8] with CMS SR7 $\left(E_{T}^{\prime}>550 \mathrm{GeV}\right)$ [7].
ATLAS quotes an expected background of $1,030 \pm 60$ events in this signal region, and finds 1,028 events. From this we compute an upper bound on the number of signal events at $95 \%$ C.L., $N_{95, \text { ATLAS }}=134$ events, to be compared with an upper bound of 146 events quoted by ATLAS. CMS expects $509 \pm 66$ events in their SR7, and observes 519 events. Our computed $N_{95, \mathrm{CMS}}=145$ whereas CMS cites a value of 142 .

Combining these SRs we have a total expected background of 1,539 \pm 89 events, whereas the actual number of observed events is 1,547. From this we compute a combined $95 \%$ C.L. upper bound on the number of signal events $N_{95 \text {,combined }}=198$, to be compared with the sum of the individual $N_{95}$ values of 279 events. The proper statistical combination thus reduces the upper bound on the total number of signal events by about $30 \%$.

In practice, we let CHECKMATE select the two signal regions which are expected to have the best sensitivity, based on the expected number of background events. We combine only these two SRs which are statistically independent since they refer to different experiments. In this way we avoid "look elsewhere" problems that could arise if we combined all nine ATLAS signal regions with all seven CMS signal regions. The most sensitive signal region depends on the value of $m_{\chi}$, which (for large mediator mass) basically fixes the kinematics of the process.

Unfortunately, we find that the combination strengthens the bound on $\Lambda$ in the model with an axial vector mediator only very slightly. For example, for light WIMPs, $m_{\chi}=1 \mathrm{GeV}$, and again only including contributions with $N_{\mathrm{NP}}=2$, we find a combined $95 \%$ C.L. lower bound $\Lambda>970 \mathrm{GeV}$, compared to individual 95\% C.L. lower bounds of $950 \mathrm{GeV}$ for ATLAS and $900 \mathrm{GeV}$ for CMS. This improvement is not really significant. 


\section{COMPARISON BETWEEN THE EFT AND SIMPLIFIED MODELS}

In this section we first discuss under what circumstances our simplified models can be accurately described by an EFT as far as monojet production is concerned, taking the finite width of the mediator into account. We then briefly discuss other limits on the models, which have nothing to do with monojet searches.

\section{A. Finite width effects and applicability of the EFT}

So far we have simultaneously chosen large masses and small widths for our $s$-channel mediators. This allows us to reproduce the EFT limit in our formalism; note that FeynRules does not allow us to directly input four-fermion operators into the Lagrangian. We also saw that the current LHC bound on the scale $\Lambda$ is about a TeV for the axial vector mediator, and only about $40 \mathrm{GeV}$ for the pseudoscalar mediator, even for light WIMPs; for heavier WIMPs the bounds become even weaker.

However, as pointed out at the end of Sec. II, requiring the mediator mass to be significantly larger than $\Lambda$ requires couplings which are larger than 1 . This in turn leads to large widths of the mediator. In other words, the combination of a large mediator mass $M^{2} \gg \Lambda^{2}$ with a small mediator width $\Gamma^{2} \ll M^{2}$ cannot be realized in a physical model.

In a more realistic situation, i.e., in a real "simplified model," the width of the mediator is instead a derived quantity [39-41]. For this discussion we consider Dirac fermionic $\chi$ of mass $1 \mathrm{GeV}$; as noted above, for heavier $\chi$ the bound on $\Lambda$ is weaker, making the problem even more severe. This means that the mediator can always decay into WIMPs as well as into quarks. ${ }^{5}$ At tree level, the widths of the pseudoscalar and axial vector mediators are given by

$$
\begin{aligned}
\Gamma_{P}= & \frac{M_{P}}{8 \pi}\left[g_{\chi P}^{2}\left(1-\frac{4 m_{\chi}^{2}}{M_{P}^{2}}\right)^{1 / 2}\right. \\
& \left.+N_{c} g^{2} \sum_{q} \frac{m_{q}^{2}}{v^{2}}\left(1-\frac{4 m_{q}^{2}}{M_{P}^{2}}\right)^{1 / 2}\right] ; \\
\Gamma_{A}= & \frac{M_{A}}{12 \pi}\left[g_{\chi A}^{2}\left(1-\frac{4 m_{\chi}^{2}}{M_{A}^{2}}\right)^{3 / 2}\right. \\
& \left.+N_{c} g_{q A}^{2} \sum_{q}\left(1-\frac{4 m_{q}^{2}}{M_{A}^{2}}\right)^{3 / 2}\right] .
\end{aligned}
$$

We have again assumed that the axial vector mediator has common coupling $g_{q A}$ to all quarks, whereas the coupling

\footnotetext{
${ }^{5}$ If the decay into WIMPs were not possible, one could generate a small width of the mediator by choosing its coupling to quarks to be very small. In order to keep $\Lambda$ fixed, the coupling strength to WIMPs would have to be increased such that the product of the couplings is constant. This quickly would require couplings to the WIMP exceeding $\sqrt{4 \pi}$, again indicating that at least one sector of the model is not perturbative.
}

of the pseudoscalar mediator to quarks is given by Eq. (6), with $y_{q} / \sqrt{2}=m_{q} / v$. The factor $N_{c}=3$ accounts for the color of quarks.

Evidently the decay width of the mediator scales like the squared coupling times the mass of the mediator. As long as the mediator is narrow, $\Gamma^{2} \ll M^{2}$, increasing $M$ for fixed $\Lambda$ implies that the couplings grow proportional to $M$; see Eqs. (4) and (8). In that case the mediator's width will scale like $M^{3}$. This means that the $M^{2} \Gamma^{2}$ term in the squared propagator of the mediator, as obtained from Eqs. (9) and (10), will scale like $M^{8}$ with increasing mass. When the mediator's width becomes comparable to its mass a perturbative treatment is no longer possible; moreover, Eqs. (4) and (8), which ignore the $\Gamma^{2} M^{2}$ term in the squared propagator, are no longer valid.

In order to illustrate this problem, consider the AV case with $M_{A}=10 \mathrm{TeV}, \Lambda=1 \mathrm{TeV}$ and $g_{\chi A}=g_{q A}$ for all six quark flavors. Equation (4) then gives $g_{\chi A}=g_{q A}=\sqrt{10}$, which via Eq. (10) leads to $\Gamma_{A} \simeq 50 \mathrm{TeV}=5 M_{A}$. This is clearly beyond the domain of perturbation theory, and beyond the domain of applicability of Eq. (4).

In our earlier analyses we chose $M_{A}=10 \mathrm{TeV}$ just to be on the safe side; for such a heavy mediator on-shell production of the mediator should clearly be negligible, and the EFT limit should be applicable to analyses of LHC data. We saw above that for fixed $\Lambda$ the mediator's width grows like the third power of its mass. It is thus important to find the minimal mass of the mediator for which the predictions of the simplified model can be reproduced accurately by the EFT.

In order to determine this we again only consider contributions with $N_{\mathrm{NP}}=2$. We compute the monojet cross section after cuts for two values of the width of the mediator, $\Gamma=1 \mathrm{GeV}$ and $\Gamma=M / 2$. In case of an axial vector mediator, contributions with initial $b$ or $t$ quarks are very small, due to their small parton densities in the proton. For a pseudoscalar mediator, Eq. (8) implies $g=g_{\chi P} \simeq 62$ for $\Lambda=40 \mathrm{GeV}$ (near the current bound) already for $M_{P}=1 \mathrm{TeV}$. Equation (6) would thus imply a coupling $g_{P t}$ to the top quark well beyond $\sqrt{4 \pi}$. For only a slightly heavier mediator, its coupling to $b$ quarks would become nonperturbative as well. We therefore set the couplings to $b$ and $t$ quarks to zero in both scenarios. The couplings of the axial vector mediator to the remaining four quarks are set equal to one another, while the couplings of the pseudoscalar mediator to these quarks are proportional to the respective Yukawa couplings; see Eq. (6).

We then compute the cross section for $\chi \bar{\chi}+$ jet(s) "monojet" events after cuts as a function of the mediator mass, keeping $\Lambda$ fixed. For $\Gamma=M / 2$, we include the width dependence of $\Gamma$; i.e., we replace $M$ by $\left(M^{4}+M^{2} \Gamma^{2}\right)^{1 / 4}$ in Eqs. (4) and (8). We fix $\Lambda$ to 900 (40) $\mathrm{GeV}$ for an axial vector (pseudoscalar) mediator, close to the current experimental limits. Since we only include contributions where a 

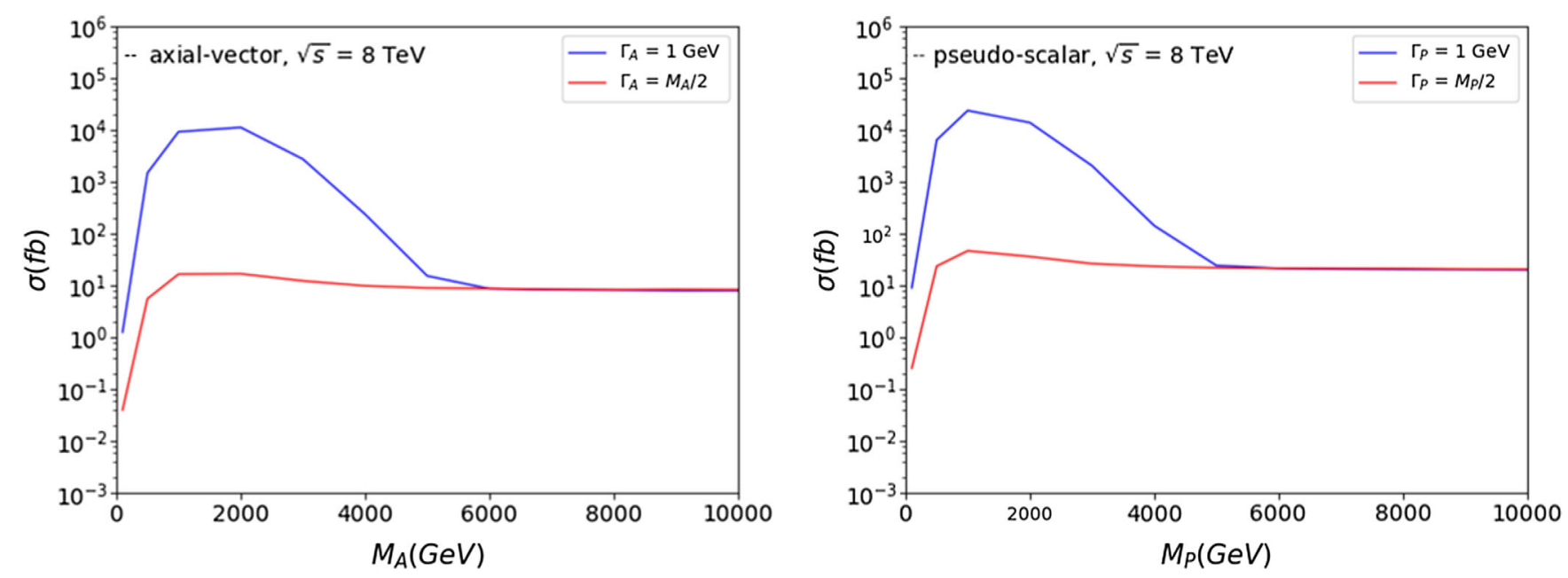

FIG. 4. Monojet cross section after cuts for two mediator widths. The left frame is for the axial vector mediator with $\Lambda=900 \mathrm{GeV}$ with cuts taken from ATLAS SR7, the right frame for the pseudoscalar mediator with $\Lambda=40 \mathrm{GeV}$ with cuts taken from ATLAS SR6. The couplings have been varied along with the mediator masses, such that the scales $\Lambda$ are kept fixed.

single mediator is exchanged and fix the widths of the mediators, the matrix element is always proportional to the product of couplings of the mediator to quarks and to WIMPs. This is true by construction in the EFT limit, but holds here even for on-shell exchange of the mediator.

The results are shown in Fig. 4. We see that if we fix the mediator's width to $1 \mathrm{GeV}$, as we did in our previous analyses, it should have a mass of at least $6 \mathrm{TeV}$ for on-shell production of the mediator to become negligible. Only for masses above this value does the cross section become independent of the mediator's mass for fixed $\Lambda$, as predicted by the EFT. This lower limit is basically the same for an axial vector and pseudoscalar mediator. ${ }^{6}$

However, taking such a small width exaggerates the problem. Since the width in this calculation is kept fixed, independent of the mass and couplings of the mediator, the cross section for on-shell production of the mediator scales like $1 / \Gamma$ after integrating over the Breit-Wigner peak. An artificially small width therefore implies an artificially large on-shell cross section. On the other hand, Fig. 4 also shows that even for $\Gamma=M / 2$, at the border of the perturbatively treatable domain, the cross section becomes approximately independent of the mass only for $M \geq 3 \mathrm{TeV}$. This roughly agrees with earlier results [18] for similar values of the mediator's width.

For the fixed product $g_{A}^{2} \equiv g_{\chi A} g_{q A}$ the total decay width of the axial vector mediator is minimized if $g_{q A}^{2}=g_{A}^{2} /(2 \sqrt{3})$, giving

$$
\Gamma_{A, \min }=\frac{M_{A} g_{A}^{2}}{\sqrt{3} \pi}=\frac{M_{A}^{3}}{\sqrt{3} \pi \Lambda^{2}} ;
$$

\footnotetext{
${ }^{6}$ These figures use the ATLAS cuts that offer the best expected sensitivity in the given model; this differs slightly, with the AV model favoring a slightly stronger cut on the missing $E_{T}$.
}

here we again assumed equal couplings $g_{q A}$ to all first and second generation quarks, and we have used Eq. (4). Requiring $\Gamma_{A}<0.5 M_{A}$ for a weakly coupled theory, and $M_{A}>3 \mathrm{TeV}$ so that monojet production at the $8 \mathrm{TeV} \mathrm{LHC}$ can be described adequately by the EFT, thus implies $\Lambda>1.8 \mathrm{TeV}$. This is "only" about a factor of 2 above the lower bound from the $8 \mathrm{TeV}$ data. Recall, however, that the signal cross section scales like $\Lambda^{-4}$; improving the bound by a factor of 2 would thus require a 16 times stronger upper bound on the signal cross section. We conclude that for parameter choices that give monojet cross sections near the upper bound, the model with axial vector mediator cannot be accurately described by an EFT, if the theory is weakly coupled, i.e., if perturbation theory is applicable.

This problem can also be illustrated using Fig. 5. This shows the monojet cross section after the same cuts, and for the same value of $\Lambda$, calculated from Eq. (4). The main difference is that $\Gamma_{A}$ has now been computed from Eq. (10), assuming $g_{\chi A}=g_{q A}$. We see that now there is no region of mediator mass where the cross section becomes independent of the mass, as one would expect in the EFT picture. The reason is that, as stated above, in this case the width grows like the third power of the mass. For the given choice of couplings, $\Gamma_{A}>M_{A}$ for $M_{A}>1.5 \mathrm{TeV}$. For larger values of $M_{A}$, the cross section drops approximately like $M_{A}^{-4}$. Of course, in this regime the theory is no longer weakly coupled, so the result is not reliable quantitatively. Note also that the situation would have been different had $\Lambda$ been an order of magnitude larger. In this case the cross section would indeed become almost independent of $M_{A}$ for some range of masses above $3 \mathrm{TeV}$; it would also be very small, several orders of magnitude below the experimental bound.

We saw above that already the early (2015) $13 \mathrm{TeV}$ data slightly strengthened the bound on $\Lambda$. However, going to 


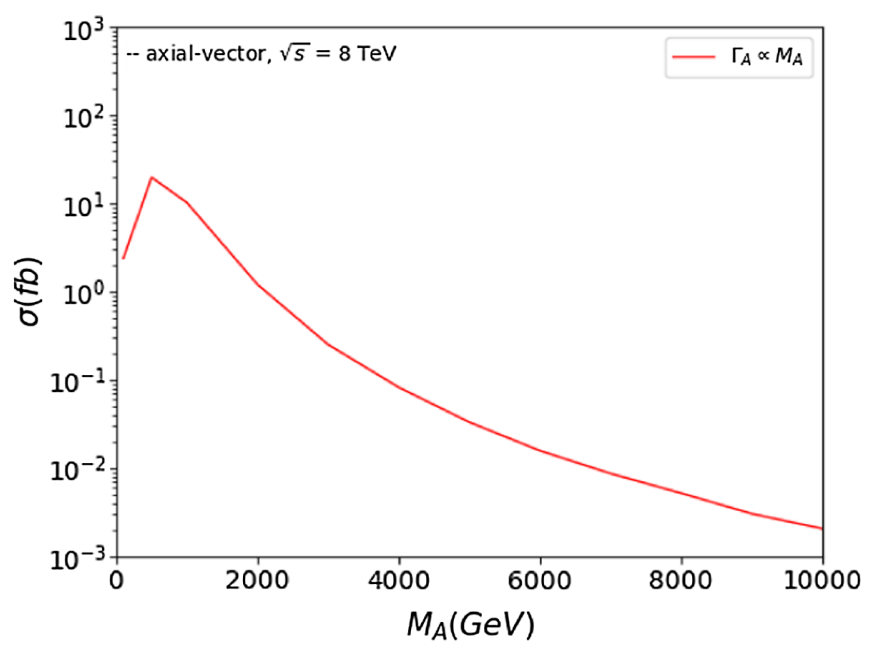

FIG. 5. Monojet cross section after cuts from ATLAS SR7 in the model with an axial vector mediator if the mediator's width is calculated from Eq. (10). Here the couplings are varied along with $M_{A}$ such that $\Lambda$ calculated using Eq. (4) is kept fixed at $900 \mathrm{GeV}$.

higher center-of-mass energy also requires higher values of $M_{A}$ for on-shell production of the mediator to be negligible, so that the theory can be approximated by an EFT for LHC purposes. For $M_{A}>5 \mathrm{TeV}, \Gamma_{A}<M_{A} / 2$ is possible only if $\Lambda>3 \mathrm{TeV}$. It seems extremely unlikely that the upper bounds on the monojet cross section at the LHC will ever become this strong.

The case of a pseudoscalar mediator is slightly different, although the conclusion will be similar. For the assumed proportionality of the coupling to a given quark to the mass of this quark, the total decay width of the mediator is dominated by decay into $c$ quarks and WIMPs. For fixed product $g_{P}^{2} \equiv g_{\chi P} g$ the total decay width is minimized if $g_{\chi P}^{2}=\sqrt{3} m_{c} g_{P}^{2} / v$, giving

$$
\Gamma_{P, \min }=\frac{\sqrt{3} M_{P}^{3} m_{c}}{4 \pi \Lambda^{3}} .
$$

Here we have again assumed that the WIMP is much lighter than the mediator, and we used Eq. (8). We again need $M_{P}>3 \mathrm{TeV}$ for the EFT to be applicable even if $\Gamma_{P}=M_{P} / 2$; see Fig. 4. Using a running charm quark mass $m_{c}\left(M_{P}\right)=0.6 \mathrm{GeV}$, we find that $M_{P}>3 \mathrm{TeV}$ and $\Gamma_{P}<M_{P} / 2$ requires $\Lambda>110 \mathrm{GeV}$. Recall that in this case the cross section scales like $\Lambda^{-6}$. Reducing the upper bound on $\Lambda$ from about 40 to $110 \mathrm{GeV}$ would thus require a reduction of the upper bound on the cross section by a factor of more than 400. As in the case of the axial vector mediator, the situation is not likely to improve very much at the $13 \mathrm{TeV}$ LHC.

Although we did not treat them explicitly, the cases with vector and scalar $s$-channel mediators are very similar to those with an axial vector and pseudoscalar mediator, as far as LHC physics is concerned (although the direct detection limits are much stronger for these cases, as noted earlier). We are thus forced to conclude that there is no weakly coupled simplified model with an s-channel mediator to which the monojet bounds derived in the EFT can be applied.

\section{B. Other constraints}

The parameters of the simplified models are also constrained by considerations not related to WIMP physics. We will be brief in this subsection, since we do not have much new to add to this discussion.

To begin with, a spin-1 particle can be described in a renormalizable theory only as a gauge boson. An axial vector mediator couples differently to the right- and lefthanded components of fermions. This can lead to anomalies. Indeed, choosing equal couplings to all quarks, and to the WIMP, gives nonvanishing anomalies [42]. These can be canceled by introducing additional fermions. However, as also pointed out in [42], these fermions cannot be much heavier than the mediator; otherwise unitarity will be violated. If these fermions can be produced in decays of on-shell mediators, its width will be even larger than assumed in the discussion of the previous subsection. Also, some LHC searches for new particles will likely lead to constraints on the masses of the mediator and the new fermions in this case. Note finally that these new fermions do not decouple; i.e., their effect might be felt also at low energies [43].

The mediator can certainly decay into $q \bar{q}$ pairs. This gives rise to a bump in the dijet invariant mass distribution. The fact that no such bump has been found leads to additional constraints on the model [44]. However, these bounds are weaker than the theoretical lower bound on the mediator mass we derived above from the requirement that monojet production at the LHC can be described by an EFT.

Finally, even if the EFT is applicable, models with $s$-channel mediators inevitably also lead to contact interactions between four quarks [45]. These have been searched for at the LHC (and earlier hadron colliders). These searches are usually interpreted in terms of quark compositeness. The resulting lower bounds on the scale $\Lambda$ are several times stronger than the ones that have been derived from monojet searches. These searches probe even larger momentum exchanges than monojet searches do. It is thus conceivable that there are model parameters where monojet searches can be interpreted as an EFT, whereas searches for "quark compositeness" cannot. If we take these constraints at face value, we would have to assume that the mediator couples much more strongly to WIMPs than to quarks; note that the coefficient of the four quark interaction operator is proportional to the square of the mediator's coupling to quarks, and does not depend on its coupling to WIMPs. 


\section{INCONSISTENCY OF THE EFT DESCRIPTION}

In this section we show that there is a purely internal inconsistency with the EFT description as used in the interpretation of monojet limits, both in the discussion of the previous sections and by the experimental groups. The point is that we have so far artificially restricted ourselves to only including diagrams where a single mediator is exchanged $\left(N_{\mathrm{NP}}=2\right.$ in the language of MadGraph). Since we need large mediator masses, and correspondingly large couplings, for the EFT to describe these contributions accurately, one may wonder whether diagrams where two mediators are exchanged $\left(N_{\mathrm{NP}}=4\right)$ might not be relevant. Here we discuss this for the axial vector mediator; similar conclusions can be derived for the pseudoscalar mediator. In the numerical results we set the mediator mass to $10 \mathrm{TeV}$, but the results would be the same for even larger masses if the couplings are chosen such that $\Lambda$ is fixed.

Here we are interested in contributions with the exchange of two mediators but without the emission of an additional parton by a QCD vertex. These contributions are therefore of lower order in QCD coupling than the $N_{\mathrm{NP}}=2$ contributions. Note that these contributions always have two partons (quarks or antiquarks) in the final state. However, we saw above that both ATLAS and CMS searches tolerate the existence of a second jet in their "monojet" searches, if certain conditions are met.

Two sample diagrams are shown in Fig. 6. In the left diagram the momentum flow through both mediators is spacelike; i.e., for our spin-1 mediator this is a vector boson fusion contribution. Note that both incoming (and outgoing) partons may be quarks, i.e., the corresponding cross section receives contributions where both incoming partons are valence quarks. This further enhances the importance of this contribution at large partonic Mandelstam- $s$. Moreover, since each mediator couples to a quark line and a WIMP line, this contribution to the scattering amplitude is of order $\left(g_{q A} g_{\chi A} / M_{A}^{2}\right)^{2}$; i.e., it can be fully described by the same $\Lambda$

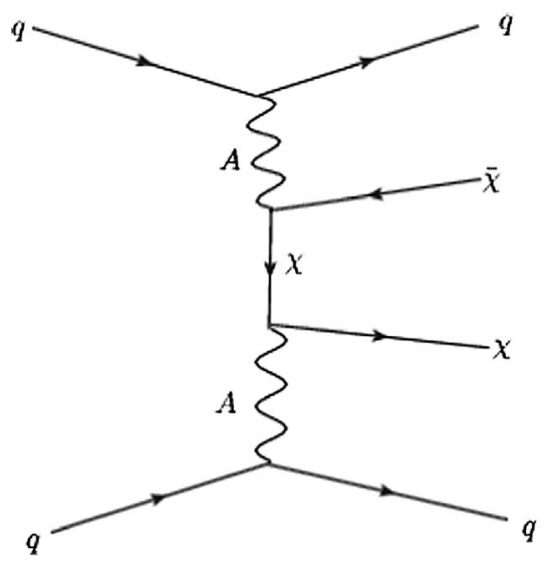

that determines the $N_{\mathrm{NP}}=2$ contributions to the cross section in the EFT limit. Finally, we note that the left diagram also exists in models with a $t$-channel mediator.

In contrast, in the right diagram one of the mediators only couples to quarks. As a result, this contribution to the matrix element is of order $g_{\chi A} g_{q A}^{3} / M_{A}^{4}$; i.e., it cannot be described only by $\Lambda$ defined in Eq. (4). In other words, these $N_{\mathrm{NP}}=4, \mathcal{O}\left(\Lambda^{-4}\right)$ contributions to the total matrix element for the signal also depend on the ratio of the two couplings, in addition to their product. Moreover, while this diagram requires exactly one antiquark in the initial state, the corresponding contribution is enhanced by a factor of the number of flavors $N_{f}$, since the flavor in the final state is not related to the flavor in the initial state.

There are additional $N_{\mathrm{NP}}=4$ contributions where the first virtual mediator "decays" into a four-fermion final state via the exchange of the second mediator; these contributions are again of order $\left(g_{q} g_{\chi} / M_{A}^{2}\right)^{2}$. All $N_{\mathrm{NP}}=4$ contributions involve the propagator of one light fermion, in addition to the two mediator propagators; e.g., the left diagram in Fig. 6 contains a $\chi$ propagator, while the right diagram contains a quark propagator. These contributions can therefore not be expressed by a local higher-dimensional operator.

In Fig. 7 we compare the bounds on $\Lambda$ that can be derived from ATLAS data for $N_{\mathrm{NP}}=2$ and $N_{\mathrm{NP}}=4$. Here we have taken equal couplings of the mediator to all quarks and to WIMPs. We see that including the $N_{\mathrm{NP}}=4$ contributions increases the upper bound on $\Lambda$ by at least 40 (150) GeV for the $\sqrt{s}=8$ (13) TeV data. An increase of $40 \mathrm{GeV}$, or about $5 \%$, does not sound very dramatic. Recall, however, that the leading contribution to the signal cross section scales like $\Lambda^{-4}$. A $5 \%$ increase of the bound on $\Lambda$ therefore corresponds to a $20 \%$ increase of the total signal cross section; at $\sqrt{s}=13 \mathrm{TeV}$, the total cross section increases by about $60 \%$.

The effect of the $N_{\mathrm{NP}}=4$ contributions becomes even more pronounced when we look at specific initial and final

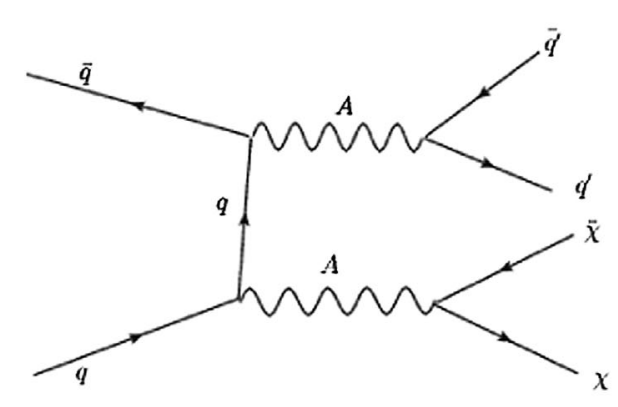

FIG. 6. Two sample Feynman diagrams that involve four vertices of the mediator, corresponding to $N_{\mathrm{NP}}=4$ in MadGraph notation. Note that there are two partons in the final state, but no QCD vertex is required. See the text for further discussion. 

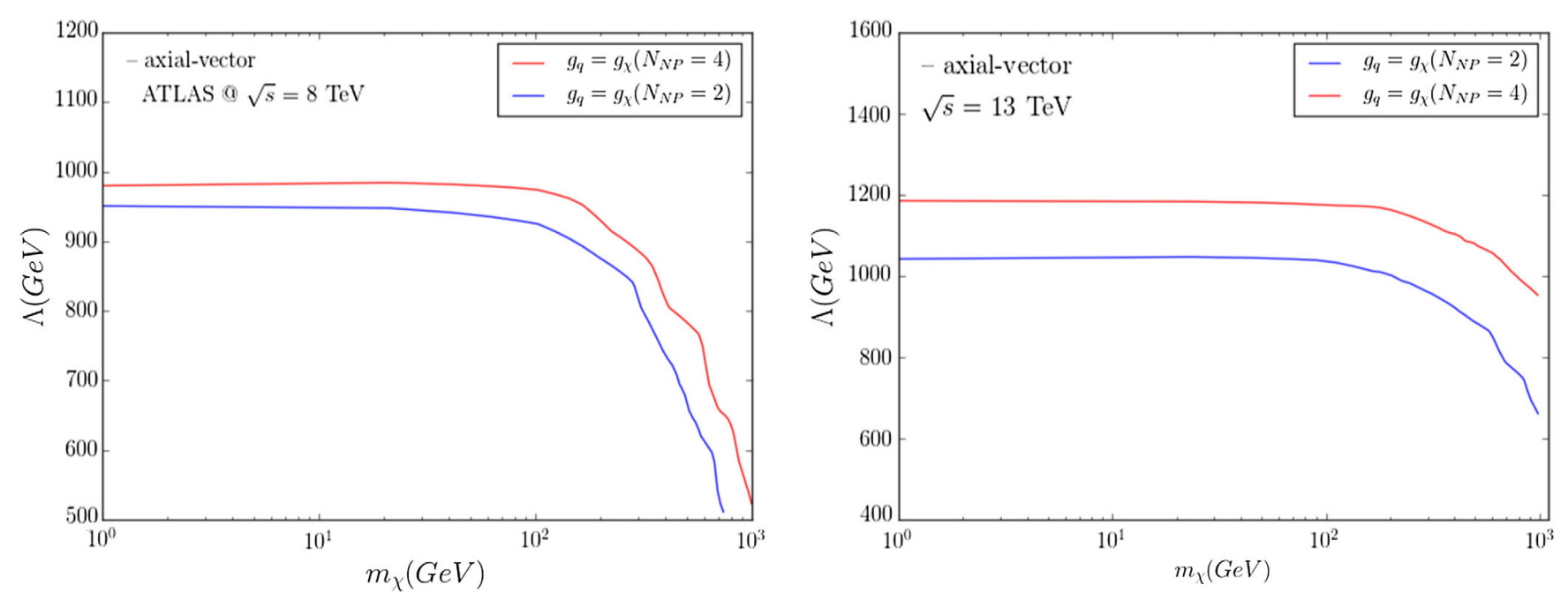

FIG. 7. Comparison of bounds on $\Lambda$ at 95\% C.L. derived from ATLAS data at $\sqrt{s}=8 \mathrm{TeV}$ (left) and 13 TeV (right). The blue curves are for $N_{\mathrm{NP}}=2$, i.e., only diagrams where a single mediator is exchanged are included, while the red curves also include diagrams where two mediators are exchanged $\left(N_{\mathrm{NP}}=4\right)$. We have set $g_{\chi A}=g_{q A}$. These results have been obtained in the EFT limit; i.e., they do not depend on the mediator mass once $\Lambda$ is fixed.

states. From the above discussion it is clear that diagrams with double mediator exchange always have two partons in the final state; they thus only contribute to the dijet part of the signal cross section, which contributes about $25 \%$ of the total cross section after matching if only the generator-level cuts are applied. Moreover, $N_{\mathrm{NP}}=4$ contributions only exist if all external partons are (anti)quarks, rather than gluons; after the generator-level cuts, for $N_{\mathrm{NP}}=2$ all-quark processes contribute about $15 \%$ to the total "dijet" cross section, or about $4 \%$ of the total signal cross section. The much stronger final ATLAS cuts enhance the importance of some of these contributions. In particular, as noted above contributions of the kind $q q \rightarrow \bar{\chi} \chi q q$ (see the left diagram in Fig. 6), are the only ones with two valence quarks in the initial state; these contributions suffer the smallest reduction of the parton densities when the energy scale of the process is increased by increasing the $E_{T}$ cut. For this particular class of initial and final states the effect of the $N_{\mathrm{NP}}=4$ contributions is very dramatic. For example, for $\Lambda=900 \mathrm{GeV}$ and $m_{\chi}=1 \mathrm{GeV}$, the $N_{\mathrm{NP}}=4$ terms increase the cross section for $u u \rightarrow u u \bar{\chi} \chi$ by a factor of 2.7 even if only the generator-level cuts are applied; here $u$ stands for a $u$ quark or antiquark. The impact of the $N_{\mathrm{NP}}=$ 4 contributions is even larger after the final cuts, since the $\Lambda^{-4}$ suppression of these matrix elements implies that they become relatively more important when the energy scale of the process is increased.

The relative importance of the $N_{\mathrm{NP}}=4$ contributions obviously increases with decreasing $\Lambda$. Since the bound on $\Lambda$ decreases with increasing WIMP mass, the impact of the $N_{\mathrm{NP}}=4$ contribution on the final bound is therefore even stronger for heavier WIMPs.
We thus conclude that for some important subprocesses contributing to the "monojet" signal including only the $\mathcal{O}\left(\Lambda_{\mathrm{AV}}^{-2}\right)$ contributions to the matrix elements underestimates the true contribution computed in our simplified model by a factor $\geq 2$. This is true even in the EFT limit, where on-shell production of the mediator is negligible.

The importance of these $\mathcal{O}\left(\Lambda^{-4}\right)$ contributions to the matrix element of the signal basically dooms the EFT description. Within our simplified model, these contributions can be computed explicitly. However, in the spirit of an EFT one would for consistency have to add all contributions of order $\Lambda^{-4}$. This includes in particular contributions from operators of mass dimension up to 8 in the effective Lagrangian, of which there are a great many. The usual treatment of ignoring all $N_{\mathrm{NP}}=4$ contributions amounts to the assumption that the coefficients of all of these dimension- 8 terms are negligible, which cannot be justified from the point of view of the EFT alone. Note that this is true already for the $\sqrt{s}=8 \mathrm{TeV}$ data. We saw above that, not surprisingly, the effect of the $N_{\mathrm{NP}}=4$ terms is even larger for the $13 \mathrm{TeV}$ data.

As a purely practical matter, the fact that $N_{\mathrm{NP}}=4$ contributions cannot be neglected means that even in the EFT limit (i.e., for large mediator mass with artificially small mediator width, and fixed $\Lambda$ ) the effective theory has nearly as many relevant parameters as the simplified model. In both cases the WIMP mass $m_{\chi}$ is obviously a free parameter. In the simplified model, one in addition needs to fix the mass of the mediator and its couplings to quarks and to WIMPs; in a true model, its width can then be calculated from Eq. (10). In the original EFT, these three parameters combine into the single parameter $\Lambda$ of Eq. (4). However, as 


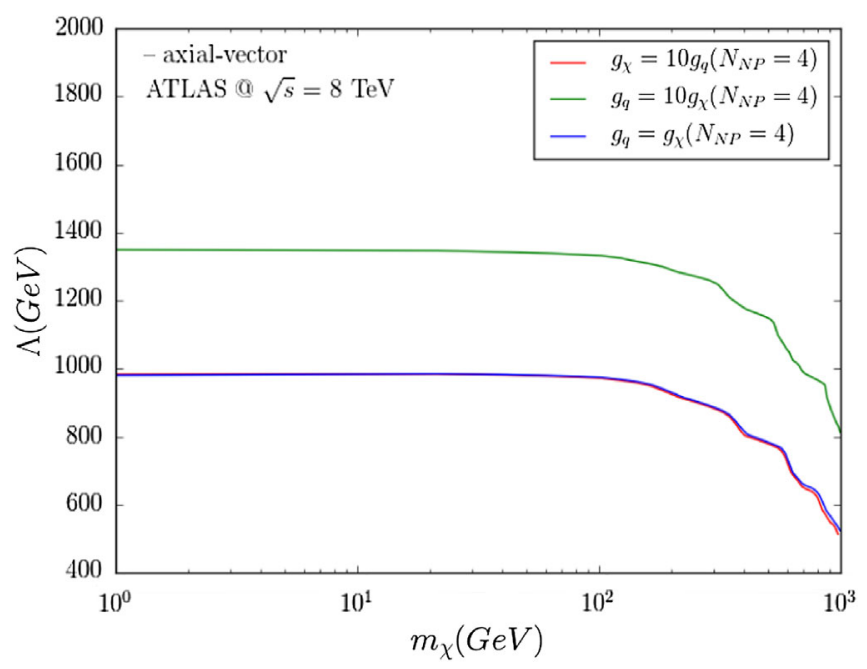

FIG. 8. Comparison of ATLAS bounds on $\Lambda$ at 95\% C.L. for $N_{\mathrm{NP}}=4 \mathrm{AV}$ interaction for different coupling ratios $g_{\chi A} / g_{q A}$.

already noted, the $N_{\mathrm{NP}}=4$ contributions in addition depend on the ratio $g_{\chi A} / g_{q A}$.

This is illustrated in Fig. 8. We see that the effect of the $N_{\mathrm{NP}}=4$ contributions becomes much more dramatic if the mediator couples more strongly to quarks than to WIMPs. For example, for $\Lambda=900 \mathrm{GeV}$ and $g_{q A}=10 g_{\chi A}$, the $N_{\mathrm{NP}}=4$ contributions increase the cross section for $u u \rightarrow$ $u u \bar{\chi} \chi$ by a factor of 120 already after the generator-level cuts. This is largely due to contributions of the type shown in the right diagram in Fig. 6. Recall that scenarios where the mediator couples more strongly to quarks are constrained very strongly by analyses of dijet data, as discussed at the end of the previous section. However, even if the mediator couples ten times more strongly to WIMPs than to quarks, the $N_{\mathrm{NP}}=4$ contributions increase the cross section for $u u \rightarrow u u \bar{\chi} \chi$ by about a factor of 2 already after the generator-level cut. Recall that $u$ here denotes either a quark or an antiquark.

We saw above that there are several classes of diagrams whose contributions scale like $\left(g_{\chi A} g_{q A}\right)^{2}$; however, without additional QCD radiation there are no $N_{\mathrm{NP}}=4$ contributions to the monojet signal that scale like $g_{\chi A}^{3} g_{q A}$. For $g_{q A}<$ $g_{\chi A}$ the $\mathcal{O}\left(g_{q A}^{3} g_{\chi A}\right)$ contributions to the matrix element become irrelevant, so that the entire $N_{\mathrm{NP}}=4$ contribution simply scales like $\Lambda^{-4}$, without additional dependence on the couplings. This explains why the total impact of the $N_{\mathrm{NP}}=4$ contributions on the bound on $\Lambda$ becomes approximately independent of the coupling ratio once $g_{\chi A} \geq g_{q A}$.

\section{SUMMARY}

In this paper we made the following original points:

(i) We quantified the lower bound on the mass of a narrow $s$-channel mediator which ensures that the
LHC monojet phenomenology can be described by an effective field theory. This lower bound is near $70 \%$ of $\sqrt{s}$, and thus much larger than the typical momentum exchange in the EFT description.

(ii) We showed that combining the $\sqrt{s}=8 \mathrm{TeV}$ data from the ATLAS and CMS collaborations increases the bound on the scale $\Lambda$ in an effective field theory (EFT) treatment of monojet production by a few percent.

(iii) We showed that there is no weakly coupled simplified model with an $s$-channel mediator that can be accurately described by an EFT, as far as the LHC monojet analyses are concerned. Here our condition for weak coupling was the requirement that the width of the mediator is less than half of its mass. The problem arises because on-shell production of the mediator becomes negligible only for masses that are considerably larger than the scale $\Lambda$ in the EFT. This requires large couplings, which in turn leads to large decay widths. This problem is even more acute for the pseudoscalar mediator.

(iv) Finally, we showed that even in an artificial EFT limit (large mediator mass with small width), the common practice of only including diagrams where a single mediator is exchanged $\left(N_{\mathrm{NP}}=2\right.$ in the language of MadGraph) underestimates the contributions of some subprocesses to the final signal by a factor of more than 2 even if only the generatorlevel cuts are imposed; the factor becomes larger for the final cuts defining the search regions that set the final limit. This shows that some contributions to the matrix element of the signal that scale like $\Lambda^{-4}$ are important. In a consistent EFT one would then have to include all relevant operators with mass dimension up to 8. Such an EFT would have many more parameters than a typical simplified model. Simply ignoring these terms is, however, not a solution, since some such terms always exist.

Our overall conclusion is that the analysis of LHC monojet data in the language of effective field theory, which was originally hoped to provide a model-independent framework, actually does not apply to any model. The EFT treatment becomes reliable only if the scale $\Lambda$ is at least a factor of 2 higher than the current bound. Since the signal cross section scales like $\Lambda^{-4}$, and the bound on this cross section at best scales like the inverse square root of the luminosity, the EFT description will probably not even be useful for the analysis of data taken at the high luminosity LHC, and certainly not in the next few years.

\section{ACKNOWLEDGMENTS}

S. B. and M. D. thank the TR33 "The Dark Universe," supported by the DFG, for support. S. B. was also supported by the Deutsche Akademische Auslandsdienst (DAAD). 
[1] M. Drees and G. Gerbier (Particle Data Group), Chin. Phys. C 40, 100001 (2016).

[2] P. A. R. Ade et al. (Planck Collaboration), Astron. Astrophys. 571, A16 (2014).

[3] G. Steigman, B. Dasgupta, and J. F. Beacom, Phys. Rev. D 86, 023506 (2012).

[4] G. Bertone, D. Hooper, and J. Silk, Phys. Rep. 405, 279 (2005).

[5] Y. Bai, P. J. Fox, and R. Harnik, J. High Energy Phys. 12 (2010) 048.

[6] M. Beltran, D. Hooper, E. W. Kolb, Z. A. C. Krusberg, and T. M. P. Tait, J. High Energy Phys. 09 (2010) 037.

[7] V. Khachatryan et al. (CMS Collaboration), Eur. Phys. J. C 75, 235 (2015).

[8] G. Aad et al. (ATLAS Collaboration), Eur. Phys. J. C 75, 299 (2015); 75, 408(E) (2015).

[9] M. Papucci, A. Vichi, and K. M. Zurek, J. High Energy Phys. 11 (2014) 024.

[10] G. Busoni, A. De Simone, T. Jacques, E. Morgante, and A. Riotto, J. Cosmol. Astropart. Phys. 09 (2014) 022.

[11] I. M. Shoemaker and L. Vecchi, Phys. Rev. D 86, 015023 (2012).

[12] P. J. Fox, R. Harnik, J. Kopp, and Y. Tsai, Phys. Rev. D 85, 056011 (2012).

[13] J. Goodman and W. Shepherd, arXiv:1111.2359.

[14] G. Busoni, A. De Simone, E. Morgante, and A. Riotto, Phys. Lett. B 728, 412 (2014).

[15] G. Busoni, A. De Simone, J. Gramling, E. Morgante, and A. Riotto, J. Cosmol. Astropart. Phys. 06 (2014) 060.

[16] D. Racco, A. Wulzer, and F. Zwirner, J. High Energy Phys. 05 (2015) 009.

[17] F. Pobbe, A. Wulzer, and M. Zanetti, J. High Energy Phys. 08 (2017) 074.

[18] O. Buchmueller, M. J. Dolan, and C. McCabe, J. High Energy Phys. 01 (2014) 025.

[19] C. Csaki, Mod. Phys. Lett. A 11, 599 (1996).

[20] S. Liem, G. Bertone, F. Calore, R. R. de Austri, T. M. P. Tait, R. Trotta, and C. Weniger, J. High Energy Phys. 09 (2016) 077.

[21] K. Cheung, P.-Y. Tseng, Y.-L. S. Tsai, and T.-C. Yuan, J. Cosmol. Astropart. Phys. 05 (2012) 001.

[22] W. Altmannshofer, P. J. Fox, R. Harnik, G. D. Kribs, and N. Raj, Phys. Rev. D 91, 115006 (2015).

[23] S. Baek, P. Ko, and J. Li, Phys. Rev. D 95, 075011 (2017).
[24] A. Alloul, N. D. Christensen, C. Degrande, C. Duhr, and B. Fuks, Comput. Phys. Commun. 185, 2250 (2014).

[25] C. Degrande, C. Duhr, B. Fuks, D. Grellscheid, O. Mattelaer, and T. Reiter, Comput. Phys. Commun. 183, 1201 (2012).

[26] J. Alwall, R. Frederix, S. Frixione, V. Hirschi, F. Maltoni, O. Mattelaer, H.-S. Shao, T. Stelzer, P. Torrielli, and M. Zaro, J. High Energy Phys. 07 (2014) 079.

[27] A. D. Martin, W. J. Stirling, R. S. Thorne, and G. Watt, Eur. Phys. J. C 63, 189 (2009).

[28] J. R. Andersen et al., arXiv:1405.1067.

[29] T. Sjostrand, S. Mrenna, and P. Z. Skands, J. High Energy Phys. 05 (2006) 026.

[30] J. Alwall et al., Eur. Phys. J. C 53, 473 (2008).

[31] M. Drees, H. Dreiner, D. Schmeier, J. Tattersall, and J. S. Kim, Comput. Phys. Commun. 187, 227 (2015).

[32] D. Dercks, N. Desai, J. S. Kim, K. Rolbiecki, J. Tattersall, and T. Weber, Comput. Phys. Commun. 221, 383 (2017).

[33] J. de Favereau, C. Delaere, P. Demin, A. Giammanco, V. Lemaître, A. Mertens, and M. Selvaggi (DELPHES 3 Collaboration), J. High Energy Phys. 02 (2014) 057.

[34] M. Cacciari, G. P. Salam, and G. Soyez, Eur. Phys. J. C 72, 1896 (2012).

[35] J. S. Kim, D. Schmeier, J. Tattersall, and K. Rolbiecki, Comput. Phys. Commun. 196, 535 (2015).

[36] M. Aaboud et al. (ATLAS Collaboration), Phys. Rev. D 94, 032005 (2016).

[37] T. Junk, Nucl. Instrum. Methods Phys. Res., Sect. A 434, 435 (1999).

[38] L. Lista, in Proceedings, 2016 European School of HighEnergy Physics (ESHEP 2016): Skeikampen, Norway, 2016 (2016), http://dx.doi.org/10.23730/CYRSP-2017-005.213.

[39] P. Harris, V. V. Khoze, M. Spannowsky, and C. Williams, Phys. Rev. D 91, 055009 (2015).

[40] M. R. Buckley, D. Feld, and D. Goncalves, Phys. Rev. D 91, 015017 (2015).

[41] J. Abdallah et al., arXiv:1409.2893.

[42] F. Kahlhoefer, K. Schmidt-Hoberg, T. Schwetz, and S. Vogl, J. High Energy Phys. 02 (2016) 016.

[43] J. A. Dror, R. Lasenby, and M. Pospelov, Phys. Rev. D 96, 075036 (2017).

[44] M. Fairbairn, J. Heal, F. Kahlhoefer, and P. Tunney, J. High Energy Phys. 09 (2016) 018.

[45] H. Dreiner, D. Schmeier, and J. Tattersall, Europhys. Lett. 102, 51001 (2013). 